ELECTRONIC RESEARCH ANNOUNCEMENTS

OF THE AMERICAN MATHEMATICAL SOCIETY

Volume 6, Pages 1-6 (January 31, 2000)

S $1079-6762(00) 00074-3$

\title{
ON SPACES WITH PERIODIC COHOMOLOGY
}

\author{
ALEJANDRO ADEM AND JEFF H. SMITH
}

(Communicated by Dave J. Benson)

\begin{abstract}
We define a generalized notion of cohomological periodicity for a connected $\mathrm{CW}$-complex $X$, and show that it is equivalent to the existence of an oriented spherical fibration over $X$ with total space homotopy equivalent to a finite dimensional complex. As applications we characterize discrete groups which can act freely and properly on some $\mathbb{R}^{n} \times \mathbb{S}^{m}$, show that every rank two $p$-group acts freely on a homotopy product of two spheres and construct exotic free actions of many simple groups on such spaces.
\end{abstract}

\section{INTRODUCTION}

A classical result in group cohomology (proved by R. G. Swan [5]) is the fact that a finite group has periodic cohomology if and only if it acts freely on a finite complex with the homotopy type of a sphere. Since then there have been numerous attempts to extend this type of result to other classes of groups, including (1) infinite groups with periodic cohomology and (2) finite groups with non-periodic cohomology.

In this note we describe a homotopy-theoretic characterization of cohomological periodicity, a notion which we now make precise.

Definition 1.1. The cohomology of a CW-complex $X$ is said to be periodic if there is an integral cohomology class $\alpha \in H^{*}(X, \mathbb{Z})$ and an integer $d \geq 0$ such that the cup product with $\alpha$ gives an isomorphism

$$
\alpha \cup-: H_{l o c}^{m}(X, \mathcal{B}) \rightarrow H_{l o c}^{m+|\alpha|}(X, \mathcal{B})
$$

for every local coefficient system $\mathcal{B}$ and every integer $m \geq d$.

Our main result is the following

Theorem 1.2. Let $X$ denote a connected $C W$-complex. The cohomology of $X$ is periodic if and only if there is an orientable spherical fibration $E \rightarrow X$ such that the total space $E$ is homotopy equivalent to a finite dimensional $C W$-complex.

Our result has a number of consequences, which we now list.

Theorem 1.3. A discrete group $\Gamma$ acts freely and properly on $\mathbb{R}^{n} \times \mathbb{S}^{m}$ for some $m, n>0$ if and only if $\Gamma$ is a countable group with periodic cohomology.

Received by the editors October 27, 1999.

2000 Mathematics Subject Classification. Primary 57S30; Secondary 20 J06.

Key words and phrases. Group cohomology, periodic complex.

Both authors were partially supported by grants from the NSF.

(C)2000 American Mathematical Society 
This represents the definitive extension and verification of a question first raised by Wall [7] for groups of finite virtual cohomological dimension (and settled in that special case in [1]).

Specializing to finite groups we show

Theorem 1.4. Let $X$ denote a finite dimensional $G-C W$ complex, $G$ a finite group, such that every abelian subgroup of the isotropy subgroups is cyclic. Then for some large integer $N>0$ there exists a finite dimensional $G-C W$ complex $Y$ with a free $G$-action such that $Y \simeq \mathbb{S}^{N} \times X$. If $X$ is finitely dominated and simply connected, then $Y$ can be taken to be a finite complex.

For finite groups with non-periodic cohomology a key problem has been to construct free actions on products of spheres. In fact given any finite group $G$, if we define its rank $r(G)$ as the dimension of its largest elementary abelian subgroup, then it has been conjectured that Swan's theorem admits the following generalization: if $k$ is the smallest integer such that a finite group $G$ will act freely on a finite complex $Y \simeq \mathbb{S}^{n_{1}} \times \cdots \times \mathbb{S}^{n_{k}}$, then $k=r(G)$. We obtain a complete answer for the rank two situation in the case of finite $p$-groups, representing the first substantial result beyond spherical space forms:

Theorem 1.5. Let $G$ denote a finite p-group. Then $G$ acts freely on a finite complex $Y \simeq \mathbb{S}^{n} \times \mathbb{S}^{m}$ if and only if $G$ does not contain a subgroup isomorphic to $\mathbb{Z} / p \times \mathbb{Z} / p \times \mathbb{Z} / p$.

Constructing interesting free actions of non-abelian finite simple groups is a particularly difficult problem. Note that they all contain a copy of $\mathbb{Z} / 2 \times \mathbb{Z} / 2$, hence cannot act freely on a single sphere. Using the classification of finite simple groups [2], character theory and local methods in homotopy theory we prove

Theorem 1.6. Let $G$ denote a rank two simple group different from $P S L_{3}\left(\mathbb{F}_{p}\right), p$ an odd prime. Then $G$ acts freely on a finite complex $Y \simeq \mathbb{S}^{n} \times \mathbb{S}^{m}$. Furthermore any such action must be exotic, i.e. it cannot be a product action.

In the following section we outline the proof of our main theorem and its consequences 11 Complete details will appear elsewhere.

\section{Proof of the Main theorem}

The proof follows from a series of lemmas which we now describe. We begin by recalling some basic notions. Cohomology will be assumed with trivial $\mathbb{Z}$ coefficients unless specified otherwise. A spherical fibration is a Serre fibration $E \rightarrow X$ such that the fiber is homotopy equivalent to a sphere $\mathbb{S}^{n-1}$. If the spherical fibration is oriented, its Euler class is the cohomology class in $H^{n}(X)$ that is the trangression in the Serre spectral sequence of the fundamental class of the fiber.

We denote the $k$ th Postnikov section of the $p$-sphere by $P_{k} \mathbb{S}^{p}$; recall that the induced map $H^{p}\left(P_{k} \mathbb{S}^{p}\right) \rightarrow H^{p}\left(\mathbb{S}^{p}\right)$ of integral cohomology groups is an isomorphism if $k \geq p$.

Lemma 2.1. Let $X$ be a connected $C W$-complex. For every integral cohomology class $\alpha \in H^{*}(X)$ of positive degree and every integer $k \geq 0$ there is an integer $q \geq 1$

\footnotetext{
${ }^{1}$ In a recent preprint [3], Mislin and Talleli have used different methods to prove a result similar to 1.3 for a large class of discrete groups, namely those which are hierarchically decomposable, with a bound on the orders of their finite subgroups.
} 
and a fibration sequence $P_{k+q|\alpha|-1} \mathbb{S}^{q|\alpha|-1} \rightarrow E \rightarrow X$ such that a generator of the integral cohomology group $H^{q|\alpha|-1}\left(P_{k+q|\alpha|-1} \mathbb{S}^{q|\alpha|-1}\right)$ trangresses to $\alpha^{q} \in H^{q|\alpha|}(X)$ in the Serre spectral sequence of the fibration.

Lemma 2.2. Let $P_{k+|\alpha|-1} \mathbb{S}^{|\alpha|-1} \rightarrow E \rightarrow X$ be a fibration sequence such that a generator of $H^{|\alpha|-1}\left(P_{k+|\alpha|-1} \mathbb{S}^{|\alpha|-1}\right)$ trangressses to $\alpha \in H^{*}(X)$ in the Serre spectral sequence. If $k \geq d-2$ and the map $\alpha \cup-: H^{m}(X) \rightarrow H^{m+|\alpha|}(X)$ is an isomorphism in integral cohomology for $m \geq d$, then there is an orientable spherical fibration $\bar{E} \rightarrow$ $X$ with Euler class $\alpha$. If in addition the map $\alpha \cup-: H_{l o c}^{m}(X, \mathcal{B}) \rightarrow H_{l o c}^{m+|\alpha|}(X, \mathcal{B})$ is an isomorphism for every local coefficient system $\mathcal{B}$ and every integer $m \geq d$, then $\bar{E}$ is homotopy equivalent to a $C W$-complex of dimension less than $d+|\alpha|$.

Lemma 2.3. Let $E \rightarrow X$ be an orientable spherical fibration, where $X$ is a $C W$ complex with $k$-skeleton $X^{(k)}$. For every integer $k \geq 0$ there is an integer $q$ such that the fibration $E_{k} \rightarrow X^{(k)}$ in the cartesian square

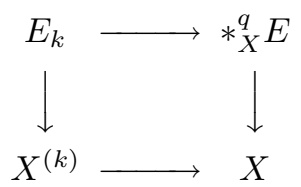

is a product fibration, where $*_{X}^{q} E$ denotes the q-fold iterated fibered join of the bundle $E \rightarrow X$.

The fiber join of a fiber bundle $F \rightarrow E \rightarrow X$ with itself produces a new fiber bundle over $X$ with fiber the join $F * F$. In particular a spherical fibration over $X$ will again yield a spherical fibration over $X$. To prove the three lemmas above we use induction combined with a systematic application of fiber joins. Roughly stated the process of fiber joins has the effect of multiplying certain relevant obstructions by an integer; on the other hand these obstructions lie in homotopy groups of spheres which have finite exponent. Hence by taking suitable fiber joins to begin with, we can ensure the vanishing of the obstruction and hence complete our inductive arguments. One sees that a proper notion of periodicity must contemplate twisted coefficients, as otherwise we cannot ensure finite dimensionality of the total space $E$. Note that for $X=B G, G$ finite, it is well known that periodicity with trivial coefficients implies our more general notion of periodicity. This should be considered an 'accidental' occurrence, as our approach makes explicit how the full notion of periodicity is required.

We now have

Theorem 2.4. Let $X$ denote a connected $C W$-complex. The cohomology of $X$ is periodic if and only if there is an orientable spherical fibration $E \rightarrow X$ such that the total space $E$ is homotopy equivalent to a finite dimensional $C W$-complex.

Proof. If $E \rightarrow X$ is an oriented spherical fibration and the total space is homotopy equivalent to a finite dimensional complex, then the Gysin sequence with local coefficients shows that $X$ has periodic cohomology.

Conversely, assume that $X$ has periodic cohomology. Let $\alpha \in H^{*}(X)$ be an integral cohomology class and $d \geq 0$ be an integer such that $\alpha \cup-: H^{m}(X) \rightarrow$ $H^{m+|\alpha|}(X)$ is an isomorphism for $m \geq d$. By Lemma 2.1, there is an integer $q \geq 1$ and a fibration sequence

$$
P_{d+q|\alpha|-1} \mathbb{S}^{q|\alpha|-1} \rightarrow E \rightarrow X
$$


such that a generator of $H^{q|\alpha|-1}\left(P_{d+q|\alpha|-1} \mathbb{S}^{q|\alpha|-1}\right)$ trangresses to $\alpha^{q} \in H^{q|\alpha|}(X)$ in the Serre spectral sequence of the fibration. By Lemma 2.2 since $d \geq d-2$, there is an orientable spherical fibration with Euler class $\alpha^{q}$, and the total space has the homotopy type of a finite dimensional complex thanks to our generalized periodicity assumption.

Let $\Gamma$ denote a discrete group; its classifying space has the homotopy type of a $\mathrm{CW}$-complex, whence we obtain

Corollary 2.5. For a discrete group $\Gamma, B \Gamma$ has periodic cohomology if and only if $\Gamma$ acts freely on a finite dimensional complex homotopy equivalent to a sphere.

If a countable discrete group $\Gamma$ acts freely on a finite dimensional complex $Y$ homotopy equivalent to a sphere, then it acts freely and properly on $\mathbb{R}^{n} \times \mathbb{S}^{m}$ for some $m, n>0$. Indeed $Y / \Gamma$ has countable homotopy groups, hence is homotopic to a countable complex which in turn is homotopic to an open submanifold $V$ in some Euclidean space; applying the h-cobordism theorem we can infer that for sufficiently large $q$ we have a diffeomorphism $\widetilde{V} \times \mathbb{R}^{q} \cong \mathbb{R}^{n} \times \mathbb{S}^{m}$ for some $m, n \geq 0$ (see [1]). Hence we obtain

Theorem 2.6. A discrete group $\Gamma$ acts freely and properly on $\mathbb{R}^{n} \times \mathbb{S}^{m}$ for some $m, n>0$ if and only if $\Gamma$ is countable and has periodic cohomology.

By a result of Wall [6], a finitely dominated CW-complex $X$ is homotopy equivalent to a finite $\mathrm{CW}$-complex if and only if its finiteness obstruction vanishes; the finiteness obstruction is an element in $\widetilde{K}_{0}\left(\mathbb{Z} \pi_{1}(X)\right)$.

Theorem 2.7. Let $X$ be a connected $C W$-complex of finite type such that the reduced projective class group $\widetilde{K}_{0}\left(\mathbb{Z} \pi_{1}(X)\right)$ is a torsion group. The cohomology of $X$ is periodic if and only if there is a spherical fibration $E \rightarrow X$ such that the total space $E$ is homotopy equivalent to a finite complex.

Corollary 2.8. Let $\Gamma$ denote a discrete group such that $B \Gamma$ is of finite type and $\widetilde{K}_{0}(\mathbb{Z} \Gamma)$ is torsion. Then $B \Gamma$ has periodic cohomology if and only if it acts freely and co-compactly on a $C W$-complex $Y$ homotopy equivalent to a sphere.

Again these results rely on the fact that fiber joins multiply obstructions by an integer - the hypothesis of finite exponent is used to dispose of the finiteness obstruction by successive fiber joins. This argument was used by Swan, based on the fact that $\widetilde{K}_{0}(\mathbb{Z} G)$ is a finite abelian group for any finite group $G$.

\section{Finite group aCtions}

Let $G$ denote a finite group; to begin we will be interested in actions of $G$ on spaces such that the isotropy subgroups have periodic cohomology. The key fact is given by

Proposition 3.1. Let $X$ denote a finite dimensional $G-C W$ complex, where $G$ is a finite group. Then the cohomology of $X \times_{G} E G$ is periodic if and only if all the isotropy subgroups have periodic cohomology.

This allows us to describe important specific situations where the results from the previous section will apply. In particular, the following theorem is a direct consequence of Theorem 2.4 and Lemma 2.3 . 
Theorem 3.2. Let $X$ denote a finite dimensional $G-C W$ complex $(G$ a finite group) such that all of its isotropy subgroups have periodic cohomology. Then there exists a finite dimensional $C W$-complex $Y$ with a free $G$-action such that $Y \simeq \mathbb{S}^{N} \times X$. If $X$ is simply connected and finitely dominated, then we can assume that $Y$ is a finite complex.

One can show that given any finite $p$-group $P$ and an element $x$ of order $p$ in its center, there exists a complex representation $V$ for $G$ such that the subgroup $\langle x\rangle$ acts freely on the associated sphere $S(V)$. In particular if $P$ is a rank two $p$-group, such a sphere will have periodic isotropy and so we can use the result above to construct a free action on a product of two spheres up to homotopy, from which we infer 1.5 .

Turning finally to the case of simple groups our strategy is again to construct an action on a sphere such that the isotropy is periodic. Unfortunately this cannot always be done, hence instead we construct a homotopy action such that the associated Borel construction has periodic cohomology. This can be done one prime at a time (using methods from local homotopy theory) and furthermore we can use local decompositions of the classifying spaces for the groups involved. Making use of the classification of finite simple groups (i.e. a case by case analysis) we show

Theorem 3.3. Let $G$ denote a rank two finite simple group other than $P S L_{3}\left(\mathbb{F}_{p}\right)$, $p$ an odd prime. Then $G$ acts freely on a finite complex $Y \simeq \mathbb{S}^{n} \times \mathbb{S}^{m}$. Furthermore any such action must be exotic, i.e. it cannot be a product action.

The last statement follows from the observation that any rank two simple group contains a copy of the alternating group $A_{4}$ and the fact that by a result due to Oliver [4] this group must act exotically.

We conclude with examples illustrating the theorem above.

Examples 3.4. Both the alternating group $A_{5}$ and the linear group $S L_{3}\left(\mathbb{F}_{2}\right)$ have complex representations of dimension three such that the action on the associated sphere has isotropy with periodic cohomology. Hence both of these groups will act freely on finite complexes of the form $\mathbb{S}^{N} \times \mathbb{S}^{5}$. On the other hand, for $G=M_{11}$, the first Mathieu group, we must use homotopy representations at the primes $p=2,3$ to construct an action on $\mathbb{S}^{383}$ giving rise to a Borel construction with periodic cohomology. Hence $M_{11}$ acts freely on a finite complex $Y \simeq \mathbb{S}^{N} \times \mathbb{S}^{383}$.

\section{REFERENCES}

[1] Connolly, F. and Prassidis, S., Groups Which Act Freely on $R^{m} \times S^{n-1}$, Topology 28, pp. 133-148 (1989). MR 90h:57052

[2] Gorenstein, D., The Classification of Finite Simple Groups, Plenum Press (1983). MR 86i:20024

[3] Mislin, G. and Talelli, O., On Groups which Act Freely and Properly on Finite Dimensional Homotopy Spheres, preprint (1999).

[4] Oliver, R., Free Compact Group Actions on Products of Spheres, Springer-Verlag LNM 763, pp. 539-548 (Arhus 1978). MR 81k:55005

[5] Swan, R.G., Periodic Resolutions for Finite Groups, Annals of Mathematics 72, pp. 267-291 (1960). MR 23:A2205

[6] Wall, C.T.C., Finiteness Conditions for CW-complexes II, Proceedings Royal Society, Series A 295, pp. 129-139 (1966). MR 35:2283

[7] Wall, C.T.C., Periodic Projective Resolutions, Proc. London Math. Soc. 39, pp. 509-533 (1979). MR 81h:18013 
Mathematics Department, University of Wisconsin, Madison, Wisconsin 53706

E-mail address: adem@math.wisc.edu

Mathematics Department, Purdue University, West Lafayette, Indiana 47907

E-mail address: jhs@math.purdue.edu 